\title{
Monitoramento tecnológico dos agentes dessensibilizantes dentinários
}

\section{Technological monitoring of dentin desensitizing agents}

\author{
Wellington Luiz de Oliveira da Rosa* \\ Adriana Fernandes da Silva** \\ Airton da Silva Oliveira ${ }^{* * *}$ \\ Rafael Guerra Lund ${ }^{* * * *}$ \\ Antônio Cesar Bortowiski Rosa Leites ${ }^{* * * *}$ \\ Evandro Piva ${ }^{* * * * *}$
}

\section{Resumo}

O uso estratégico das informações científicas e tecnológicas referentes aos dessensibilizantes dentinários pode possibilitar oportunidades de projetos científico-tecnológicos em consonância com políticas públicas de estímulo à inovação. Objetivo: Realizar um monitoramento tecnológico na área de dessensibilizantes a fim de obter um panorama que possibilite analisar o contexto atual e obter oportunidades para prospecção tecnológica. Materiais e método: Foi realizada uma busca sistemática nas bases de dados de patentes americana (USPTO), japonesa (JPO), brasileira (INPI) e europeia (EPO). Adicionalmente, o sistema Questel Orbit foi utilizado como fonte de consulta. A informação científica foi obtida das bases de dados PubMed, Scopus, Web of Science e Scielo. Foram selecionados artigos e documentos patentários referente a dessensibilizantes do período entre janeiro de 1965 e outubro de 2013. Para montagem e revisão do banco de dados, os softwares Questel Orbit e Microsoft Office Excel 2010 foram utilizados. Resultados: 261 patentes e 728 artigos satisfizeram os critérios de inclusão. Os Estados Unidos detêm 51\% da prioridade de patentes, seguido pelo Japão com 20\%. Além disso, os principais autores dos artigos não estão prospectando nessa tecnologia, uma vez que não houve relação entre eles e os principais inventores das patentes. Conclusões: Foi possível obter um panorama do setor de dessensibilizantes, o qual demonstra uma baixa apropriação tecnológica brasileira nessa indústria odontológica. A consolidação de políticas públicas de estímulos a projetos entre instituições científicas e tecnológicas, em áreas de fronteira de conhecimento ainda pouco exploradas, são alternativas estratégicas para uma presença efetiva da indústria brasileira nesse setor de mercado.

Palavras-chave: Dessensibilizantes dentinários. Indicadores de patentes. Materiais dentários.

\section{Introdução}

Os dessensibilizantes dentinários são produtos do setor odontológico que podem ser encontrados tanto para aplicação profissional como doméstica ${ }^{1,2}$, A prevalência da hipersensibilidade, que varia de 4 a $57 \%$ da população ${ }^{1}$, pode ser utilizada como parâmetro para dimensionar o amplo mercado desse setor para a indústria. Enquanto isso, a indústria de produtos médicos, odontológicos, hospitalares e de laboratórios teve um faturamento de 10 bilhões de reais em 2011 segundo a Abimo (Associação Brasileira da Indústria de Artigos e Equipamentos Mé-

Aluno de graduação. Faculdade de Odontologia (UFPel), Pelotas/RS. Brasil.

Professor adjunto, Departamento de odontologia restauradora. Faculdade de Odontologia (UFPel), Pelotas/RS. Brasil.

Aluno de mestrado em Engenharia de Materiais. Faculdade de Engenharia de Materiais (UFPel), Pelotas/RS. Brasil.

Professor adjunto, Departamento de odontologia restauradora. Faculdade de Odontologia (UFPel), Pelotas/RS. Brasil.

***** Professor adjunto, Departamento de Morfologia, Instituto de Biologia, Faculdade de Odontologia (UFPel). Pelotas/RS. Brasil.

* Professor adjunto, Departamento de Odontologia Restauradora. Faculdade de Odontologia (UFPel). Pelotas/RS. Brasil. 
dicos, Odontológicos, Hospitalares e de Laboratórios), sendo que o único segmento superavitário do mesmo ano foi o de odontologia, com saldo positivo de 6,3 milhões de dólares. ${ }^{3}$ Embora o mercado interno desses insumos para saúde tenha crescido, a grande maioria dos materiais para essas aplicações são importados e majoritariamente com proteção industrial de prioridade externa.

De acordo com a Organização Mundial de Propriedade Industrial (Ompi), mais de $70 \%$ das informações tecnológicas disponíveis somente podem ser encontradas nos documentos de patentes ${ }^{4,5}$. O "estado da técnica" contido nesses documentos proporciona o monitoramento de oportunidades para licenciamento de produtos, a redação de projetos tecnológicos para obtenção de financiamentos ou parcerias (universidade-empresa), o monitoramento de concorrentes, entre diversas aplicações ${ }^{6-9}$.

Os produtos disponíveis para o tratamento da hipersensibilidade visam essencialmente à obliteração dos túbulos dentinários e/ou à despolarização das fibras nervosas da polpa ${ }^{10-13}$. De acordo com a Teoria da Hidrodinâmica, o aumento do fluxo do fluido presente no interior dos túbulos dentinários leva a sensibilidade dolorosa ${ }^{14}$. Além dos dentifrícios dessensibilizantes de uso continuado, existem ainda tratamentos profissionais através da aplicação de materiais selantes, vernizes, resinas, ionômeros de vidro, laser, géis de fluoreto de sódio, entre outros ${ }^{2,15-17}$. Contudo, nenhum desses materiais foi considerado ideal para o tratamento da hipersensibilidade, pois com o tempo as substâncias são perdidas na cavidade oral e ocorre recidiva da dor nos dentes tratados ${ }^{11,18}$.

A coleta de informações científicas e tecnológicas referentes aos dessensibilizantes pode possibilitar uma análise estratégica e de natureza prospectiva do setor. Dessa forma, o objetivo desse estudo foi realizar um monitoramento tecnológico (estado da arte e estado da técnica) na área de dessensibilizantes dentinários a fim de obter um panorama que possibilite visualizar oportunidades para a prospecção tecnológica no Brasil.

\section{Materiais e método}

\section{Monitoramento de patentes}

Para a obtenção de patentes referentes à dessensibilizantes dentinários, realizou-se uma busca sistematizada nas bases de dados de autoridades europeia (EPO), americana (USPTO), japonesa (JPO) e brasileira (INPI). De forma complementar, foi realizada uma busca e análise desses documentos pelo sistema on-line Questel Orbit (Paris, França), que contém dados e informações em mais de 90 autoridades patentárias.
$\mathrm{Na}$ estratégia de busca empregada na base do INPI utilizou-se os seguintes termos: "dessensibilizantes dentinários", "dentifrícios dessensibilizantes", "hipersensibilidade dentinária", "anti-hiperestésico dentinário", "sensibilidade dentária" e suas variações no campo "título" e "resumo". No sistema do Questel Orbit (Paris, França) e nas bases americana, europeia e japonesa a busca foi feita empregando-se as respectivas palavras-chave em inglês: "desensitizing dentin", "desensitizing agents", "desensitizing dentifrice", "sensitivity dental", "desensitizer teeth", "hypersensitive teeth". Também foi realizada busca a partir do Código Internacional de Patentes (CIP) pelos seguintes códigos: A61K (Preparações para finalidades médicas, odontológicas e higiênicas), A61Q 11/00 (Preparações para tratar os dentes, a cavidade oral ou dentaduras, como dentifrícios e enxaguatórios orais), A61K 6/00 (Preparações para odontologia).

Como critérios de inclusão, foram selecionadas patentes relacionados à dessensibilizantes a partir de janeiro de 1965. Esse critério foi baseado no ano da introdução do conceito da Teoria da Hidrodinâmica ${ }^{19,20}$, até hoje a mais aceita para explicar o mecanismo da sensibilidade da dentina ${ }^{21-23}$. Essa teoria ainda norteia o desenvolvimento dos principais produtos para o tratamento da hipersensibilidade, uma vez que os materiais visam essencialmente à obliteração dos túbulos e/ou despolarização das fibras nervosas para o controle da dor.

\section{Revisão sistemática nas bases de artigos}

As informações científicas foram obtidas por meio dos artigos publicados desde 1965 nas seguintes bases de dados: MedLine (PubMed), Web of Science, Scielo e Scopus. Os termos de busca utilizados foram "desensitizing dentin", "desensitizing agents", "dental sensitivity", "desensitizer teeth", "hypersensitive teeth", "dentin sensitivity" e suas variações nos campos referentes à "título", "resumo" $e$ "palavra-chave".

Como critérios de inclusão, foram selecionados estudos clínicos e laboratoriais relacionados à dessensibilizantes publicados entre janeiro de 1965 e outubro de 2013. Artigos com revisões de literatura e estudos envolvendo dessensibilizantes relacionados a clareamento dental, sem definição clara de hipersensibilidade dentinária e escritos em uma língua diferente do inglês, espanhol ou português foram excluídos dessa revisão.

\section{Análise de dados}

Após remoção de duplicatas, foi feita a triagem manual de todos os documentos a partir da leitura do título e resumo. Somente os documentos que satis- 
fizeram os critérios de inclusão foram selecionados para análise do documento completo. Os dados foram tabulados em uma planilha no formato do software Microsoft Office Excel 2010 (Microsoft Corporation, Redmond, Washington, EUA) que continha os documentos do estudo organizados por; o título das patentes e dos artigos, o(s) nome(s) do(s) depositante(s) ou autor(es), o(s) nome(s) do(s) inventor(es), a data de depósito da patente ou publicação do artigo, o status do documento, o(s) país(es) de prioridade, o(s) mecanismo(s) de ação do(s) dessensibilizante(s), a(s) base(s) de patente(s) pesquisada(s) e a presença ou a ausência de PCT. Adicionalmente, para os documentos de patentes o sistema Questel Orbit foi utilizado para análise de dados.

\section{Resultados}

Inicialmente um total de 883 patentes foi encontrado. Dessas, 550 (62\%) foram excluídas após leitura do título e resumo (por não conformidade com o escopo do estudo) ou após leitura completa dos documentos (por não se enquadrarem nos critérios de inclusão). Além disso, 72 eram duplicatas (Figura 1). Somente 261 patentes $(30 \%)$ foram incluídas na análise. A Figura 2 mostra a porcentagem de patentes encontradas por base sem a remoção de duplicatas, uma vez que há patentes depositadas em mais de uma base.

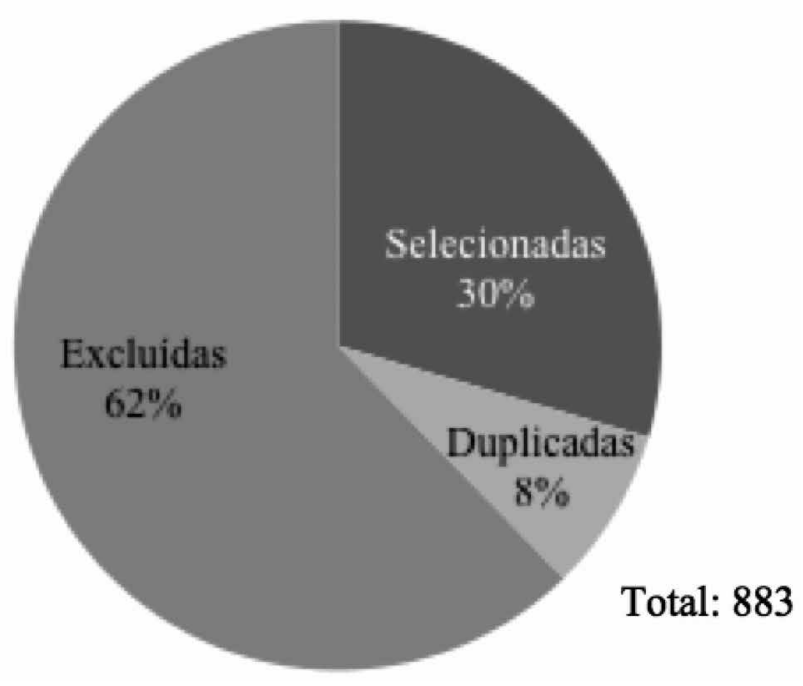

Figura 1 - Processo de seleção das patentes: documentos excluídos (550), duplicados (72) e selecionados (261) para o estudo

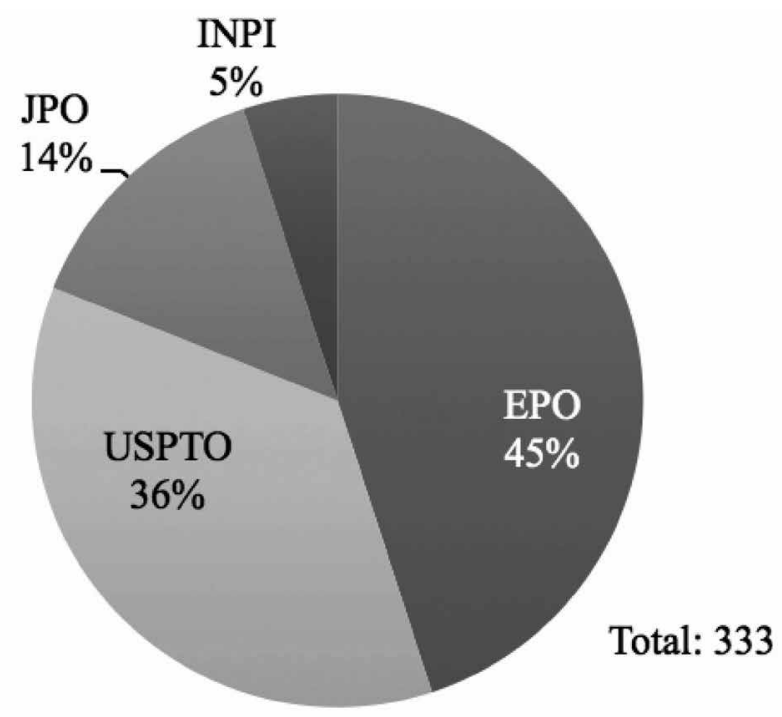

Figura 2 - Ilustração gráfica da porcentagem de patentes encontradas por base de dados (sem remoção de duplicatas)

Enquanto isso, nas bases de artigos encontrou-se 6667 estudos, sendo que 5553 (83\%) foram excluídos por não conformidade com esse estudo. Além disso, 386 (6\%) foram removidos por duplicidade (Figura 3). Desta forma, 728 (11\%) artigos preencheram todos os critérios de inclusão para análise. Os dados e informações obtidos dos 728 artigos e 261 patentes foram utilizados na análise científica e tecnológica dos materiais dessensibilizantes.

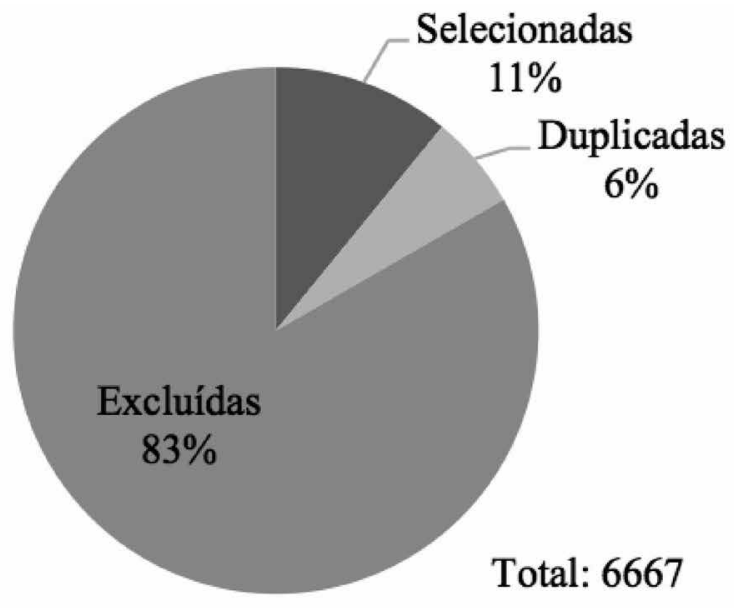

Figura 3 - Processo de seleção dos artigos: documentos excluídos por não conformidade do título e/ou resumo (5553), duplicados (386) e selecionados para o estudo (728)

\section{Discussão}

Com base nos dados bibliográficos das patentes e artigos referentes a dessensibilizantes dentinários foi possível elaborar um diagnóstico sobre o estágio de desenvolvimento desta tecnologia, sobre a proteção patentária desse material odontológico e as áreas estratégicas ainda pouco protegidas que 
podem ser exploradas. Houve um aumento gradual de informação científica e tecnológica sobre o tema, com uma maior quantidade de publicações de artigos em praticamente todos os períodos analisados, exceto em 1999 (Figura 4). O fato dos pedidos de patente permanecem em fase de sigilo durante 18 meses após o depósito é um dos aspectos limitante em qualquer análise tecnológica. Dessa forma os depósitos posteriores a abril de 2012 não puderam ser resgatados.

Embora haja muito mais informações científicas que tecnológicas disponíveis nesse setor, o resgate de dados apenas dos artigos científicos poderia demonstrar um quadro distorcido da área. $\mathrm{O}$ tratamento dessas informações é a base do processo de monitoramento tecnológico ou, de um modo mais geral, da inteligência competitiva, que ocorre quando a informação é analisada para a competitividade. ${ }^{5} \mathrm{E}$ necessária uma maior apropriação das informações científicas por parte do setor produtivo para que o desenvolvimento de novas tecnologias responda às políticas públicas de estímulo a inovação.

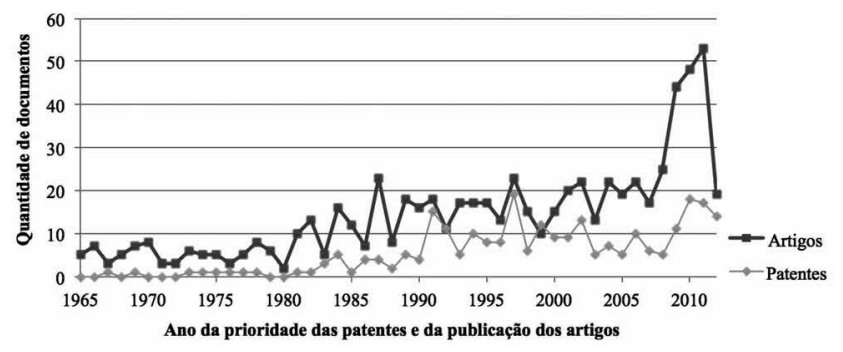

Figura 4 - Evolução anual do número de pedidos de patentes e artigos referentes à dessensibilizantes dentinários (19652012)

Com relação ao mecanismo de ação dos dessensibilizantes (Figura 5), 69\% das patentes depositadas são de produtos que visam somente à obliteração dos túbulos dentinários, enquanto $13 \%$ têm ação de despolarização das fibras nervosas. Já as patentes que incluem dispositivos usados no tratamento da hipersensibilidade (na aplicação de dessensibilizantes ou mesmo escovas dentais) estão entre os outros 4\%. Uma vez que tanto a ação de obliteração como de despolarização é temporária ${ }^{11,18}$, pode-se considerar que nenhum produto patenteado resolve definitivamente o problema da hipersensibilidade.

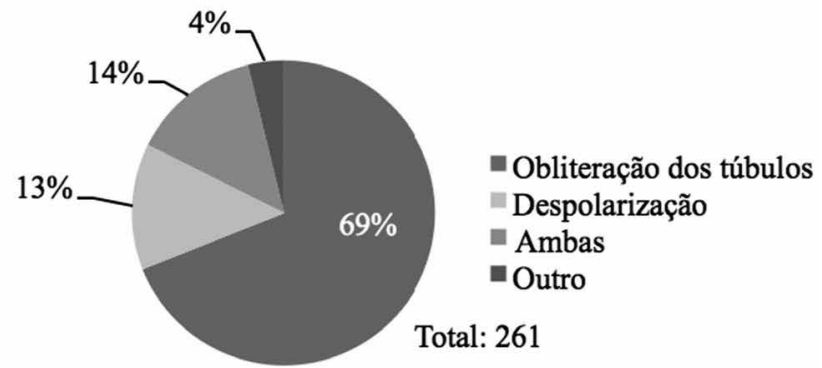

Figura 5 - Relação das patentes de acordo com o mecanismo de ação dos dessensibilizantes dentinários
A fim de analisar os países com desenvolvimento tecnológico mais expressivo no setor, foi analisado o "país de prioridade" dos documentos de patentes, que representa o local onde foi efetuado o primeiro depósito da tecnologia (Figura 6). Os Estados Unidos detêm $51 \%$ da prioridade, seguidos pelo Japão com $20 \%$. Já o Brasil possui apenas $1 \%$ da prioridade, o que indica uma deficiência do país em pesquisa e desenvolvimento tecnológico nessa área.

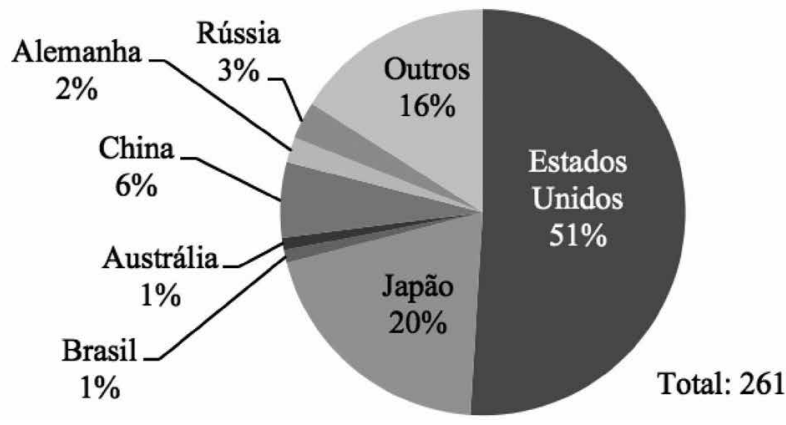

Figura 6 - Distribuição dos países com prioridade dos pedidos de patente

Para identificar os principais mercados para a comercialização desses produtos pode-se usar como parâmetro os países em que mais foram depositadas patentes referentes à tecnologia (Figura 7). Novamente há uma predominância de depósitos no mercado norte-americano. Enquanto isso, 29\% dos artigos publicados da área também tem origem americana (Figura 8). Muitos dos países que mais realizaram estudos referentes a esse material não estão entre os que mais patentearam a tecnologia (Figuras 6 e 8). No Brasil, por exemplo, o desenvolvimento tecnológico possivelmente não condiz com o que é investido em pesquisas no setor, uma vez que o país publicou cerca de 5\% dos estudos e detém apenas $1 \%$ da prioridade de patentes. Usando esse estudo de caso dos dessensibilizantes e com os devidos cuidados de extrapolação, é possível afirmar que esse setor provavelmente segue uma tendência de outras tecnologias em saúde, demonstrando que Brasil é um país em que a cultura da proteção industrial ainda não está suficientemente difundida. 


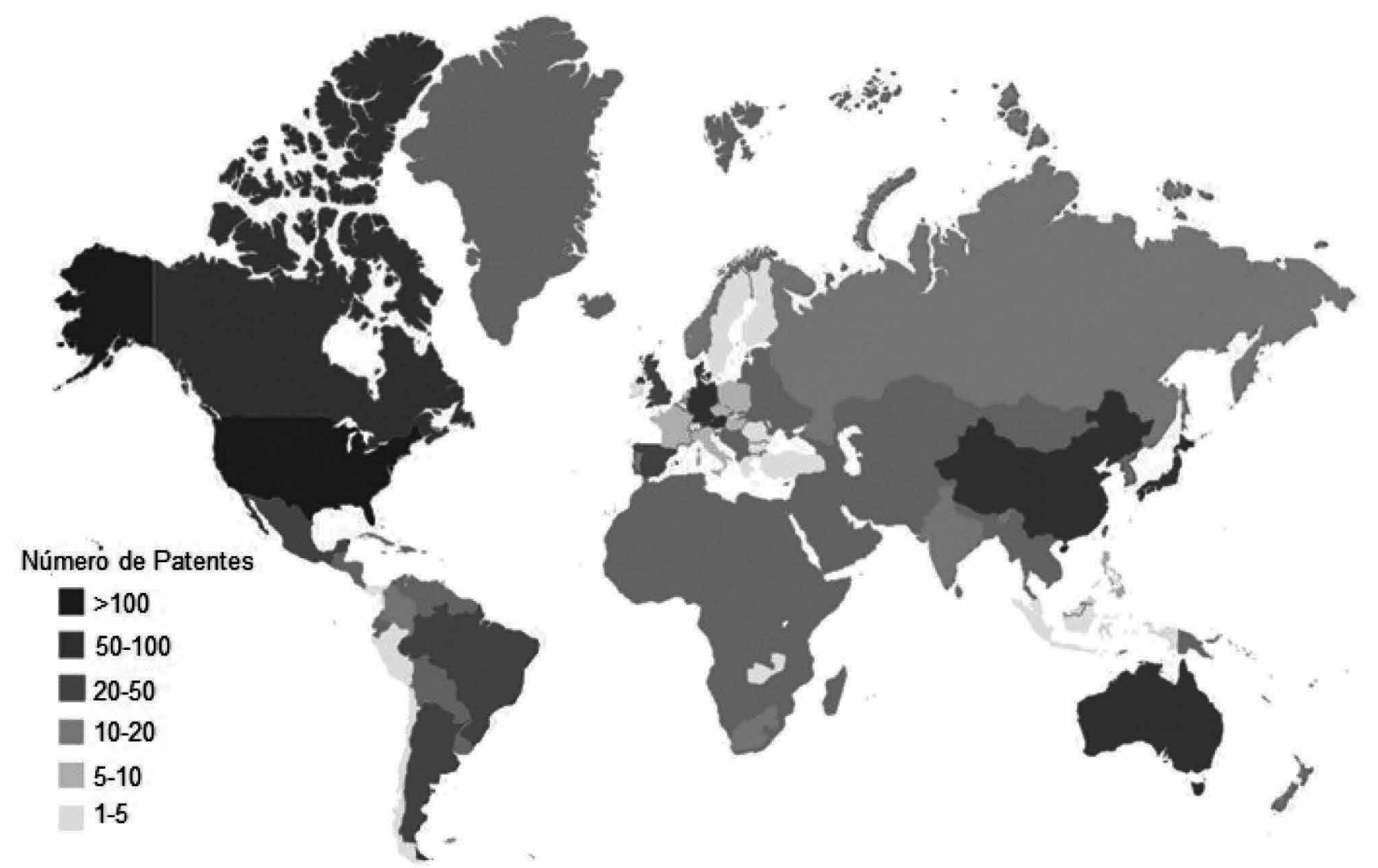

Fonte: Questel Orbit. Acesso em outubro de 2013.

Figura 7 - Número de patentes referentes à dessensibilizantes dentinários depositados no mundo de janeiro de 1965 a outubro de 2013

Foram encontradas informações quanto ao status do pedido em 67 documentos de patentes, sendo que $42 \%$ desses já haviam expirado. A avaliação da situação atual patentária tem se mostrado uma interessante estratégia de ampliação de mercado e melhoria da competitividade ${ }^{6}$. Após o período de privilégio, o invento cai em domínio público, o que pode derivar em novas apropriações do conhecimento tecnológico, com investimentos menores dos que os praticados na patente original, além de proporcionar subsídios a futuros projetos científicos ${ }^{6}$.

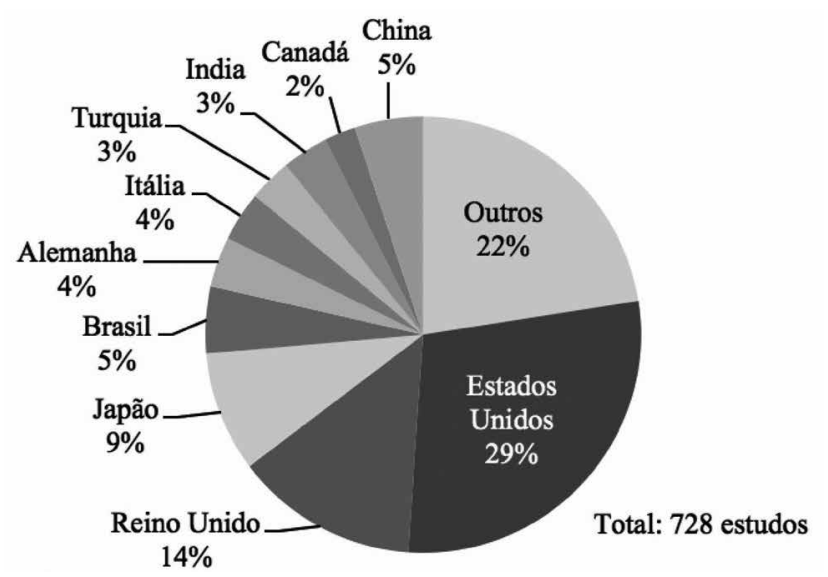

Figura 8 - Distribuição dos países com artigos publicados referentes a dessensibilizantes dentinários
As principais empresas e centros de pesquisa que tem trabalhado nesse setor (Figura 9) são a Colgate-Palmolive Company (Nova York, NY, EUA) e a GlaxoSmithKline (Londres, Reino Unido). Essas informações permitem identificar quem tem desenvolvido produtos na área, sendo importante para universidades, para centros de pesquisa ou para pesquisadores independentes que buscam parcerias para o desenvolvimento de novos dessensibilizantes. Deve-se ainda levar em consideração que as empresas não apenas patenteiam para proteger suas tecnologias, mas também para usá-las em futuras ações estratégicas como no bloqueio de entrada de produtos dos concorrentes ${ }^{24}$. A transferência de tecnologia envolve ainda o conhecimento das universidades que é transferido para as empresas, para que a indústria possa investir no desenvolvimento de novos produtos e processos ${ }^{8}$.

A cadeia de inovação pode ser definida como a sequência de eventos que incluem gastos com pesquisa e desenvolvimento, proteção da propriedade intelectual e lançamento de novos produtos e/ou serviços no mercado ${ }^{25}$. Ao analisar os dez principais autores dos artigos e inventores das patentes referentes a dessensibilizantes (Figuras 10 e 11), verifica-se que não ocorreu relação entre eles. Mesmo havendo relação entre o conhecimento científico e tecnológico, os autores que mais publicam artigos e detêm o conhecimento científico do setor não são os principais inventores que estão desenvolvendo pro- 
dutos e os patenteando para a inovação e prospecção da tecnologia.

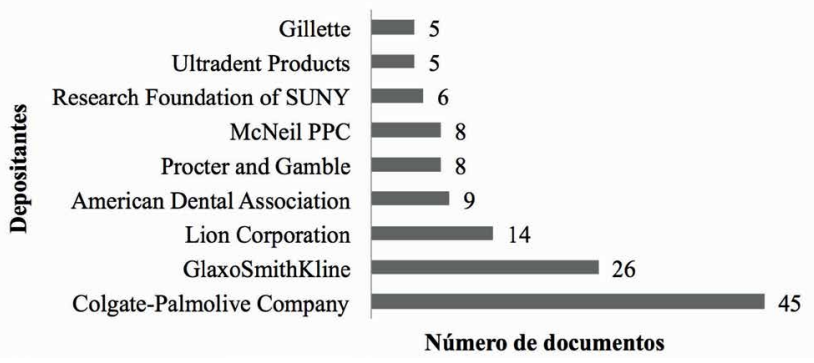

Figura 9 - Número de patentes referentes a agentes dessensibilizantes depositadas pelos principais depositantes

Como limitação deve ser reconhecida que as buscas em bancos de patente não abrangem documentos ainda na fase de sigilo (18 meses após o depósito do documento). Por causo disso, há a necessidade de ações contínuas de monitoramento em qualquer setor tecnológico. Sistemas online como o Questel Orbit (Paris, França) e softwares como VantagePoint (Search Technology Inc., Norcross, GA, EUA) facilitam esse processo, uma vez que permitem a busca e a análise de patentes em diversas autoridades simultaneamente. Contudo, o uso de tais recursos exige custo e treinamento adequado. Além disso, os desenvolvimentos científicos e tecnológicos são resultantes de interações complexas entre diferentes fatores, que envolvem trajetórias tecnológicas em evolução e competição, visões de futuro conflitantes, necessidades sociais muitas vezes urgentes e políticas públicas de estímulo a empresas emergentes e a inovação ${ }^{26}$. Também deve ser levado em consideração que o patenteamento pode não ser o único meio para a proteção tecnológica. Vantagens de segredo industrial podem ser de grande valia para as empresas, o que implica que a análise tecnológica por meio de patentes pode levar a um quadro distorcido das atividades inovadoras da indústria ${ }^{24}$.

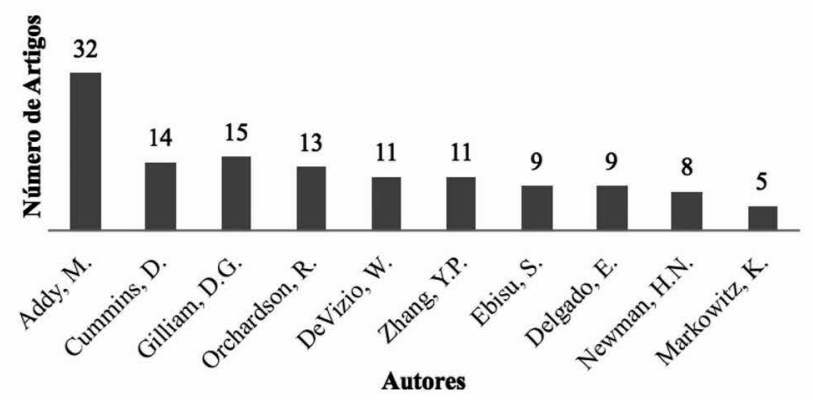

Figura 10 - Número de artigos publicados pelos 10 principais autores

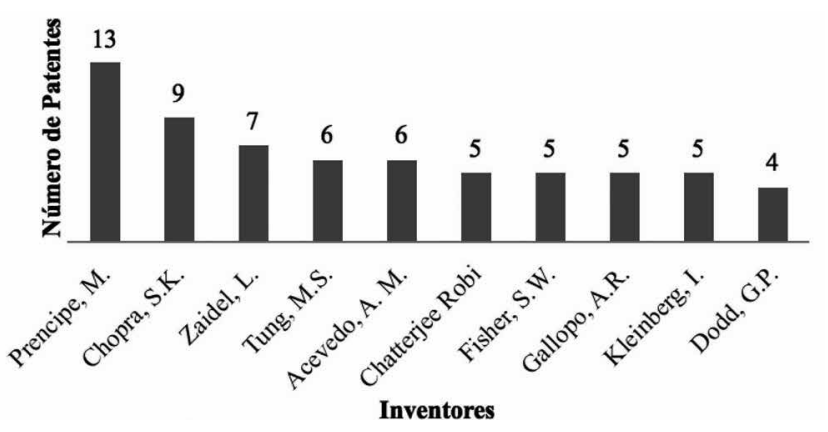

Figura 11 - Número de patentes depositadas pelos 10 principais inventores
A partir dessa análise puderam ainda ser observadas oportunidades de desenvolvimento no setor, que investe mais em produtos com substâncias para obliterar os túbulos dentinários e/ou despolarizar as fibras nervosas da polpa. A informação científica mostra que esses materiais têm efeitos apenas temporários, uma vez que com o tempo as substâncias são perdidas para a cavidade oral. Por causa disso, deve-se procurar investir em terapias alternativas para o tratamento, responsáveis por apenas $4 \%$ das tecnologias atualmente protegidas (Figura 5). Entre as novas tecnologias potencialmente inovadoras destaca-se as concebidas nas áreas de fronteira de conhecimento, como a nanotecnologia e biotecnologia, ainda pouco exploradas e protegidas para o setor de proteção abordado no presente estudo. Além disso, a compreensão e o aproveitamento do potencial biológico de reparo dentinário podem ser explorados em projetos científico-tecnológicos para o desenvolvimento de novos materiais dessensibilizantes. Ações integradas entre instituições científicas, tecnológicas e o setor produtivo devem ser estimuladas, bem como os financiamentos a tecnologias emergentes em âmbito de políticas públicas de inovação. Dessa maneira poderá ser possível aumentar tanto a projeção científica do Brasil como sua representatividade em apropriação de tecnologias da área de saúde.

\section{Conclusões}

Muitos dos países que mais realizaram estudos referentes à dessensibilizantes dentinários não estão entre os que mais patentearam a tecnologia. Deve-se procurar investir em pesquisa e desenvolvimento em áreas poucos exploradas e promissoras, como no caso das terapias biológicas e nanotecnologia. Também merece atenção o fato de que há muita informação disponível apenas em patentes que não é pesquisada e analisada. A consolidação de políticas públicas de estímulos a projetos entre instituições científicas e tecnológicas, em áreas de fronteira de conhecimento ainda pouco exploradas, são alternativas estratégicas para uma presença efetiva da indústria brasileira neste setor de mercado.

\section{Agradecimentos}

Ao CNPq pela concessão da bolsa PIBITI (Processo 155168/2012-6), a Coordenadoria de Inovação Tecnológica da UFPel (CIT/UFPel), ao Centro de Desenvolvimento e Controle de Biomateriais (CDC-Bio/UFPel). 


\section{Abstract}

The strategic use of scientific and technological information related to dentin desensitizing agents may allow opportunities for scientific and technological projects in accordance with public policies to stimulate innovation. Objective: To perform a technological monitoring of dentin desensitizing agents in order to obtain an overview that allows analyzing the current context, and to obtain opportunities for technological prospecting. Materials and method: A systematic search was performed on American (USPTO), Japanese (JPO), Brazilian (INPI), and European (EPO) patent databases. Additionally, the Questel Orbit system was used as reference source. Scientific information was obtained from PubMed, Scopus, Web of Science, and Scielo databases. Studies and patents from January 1965 to October 2013 relating to desensitizing agents were selected. Questel Orbit and Microsoft Office Excel 2010 software were used to build and review the database. Results: A total of 261 patents and 728 articles fulfilled the inclusion criteria. According to countries with priority in patents, the United States holds 51\%, followed by Japan with $20 \%$. Moreover, the main authors of the articles are not prospecting in this technology, since there was no relationship between them and the main inventors of the patents. Conclusion: It was possible to obtain an overview of the dentin desensitizing agents sector, which shows a low technological appropriation of this dental industry by Brazil. The consolidation of public policies to stimulate projects between scientific and technological institutions in poorly explored knowledge boundaries, are strategic alternatives for an effective presence of the Brazilian industry in this market sector.

Keywords: Dentin desensitizing agents. Patent indicators. Dental materials.

\section{Referências}

1. Orchardson R, Gillam DG. Managing dentin hypersensitivity. J Am Dent Assoc 2006; 137(7):990-8; quiz 1028-9.

2. Sgolastra F, Petrucci A, Gatto R, Monaco A. Effectiveness of laser in dentinal hypersensitivity treatment: a systematic review. J Endod 2011; 37(3):297-303.

3. Associação Brasileira da Indústria de Artigos e Equipamentos Médicos O, Hospitalares e de Laboratórios (ABIMO). ABIMO divulga Estudo Setorial. 2012 [citado 2012 Mai 22]. Disponível em URL: http://www.abimo.org.br/modules/news/ article.php?article_id $=286$

4. Ferraz MCC. Reflexões sobre o Uso de Documentos de Patentes em Cursos de Graduação. Revista Brasileira de Inovação 2008; 7(2):287-312.

5. Ortiz LC, Ortiz WA, Ortiz WA. Ferramentas alternativas para monitoramento e mapeamento automatizado do conhecimento. Ci Inf, Brasília 2002; 31(3):66-76.

6. Canongia C, Pereira MdNF, Antunes A. Gestão da informação e monitoramento tecnológico: o mercado dos futuros genéricos. Perspect cienc inf 2002; 7(2):155-66.
7. Haase H, Araújo ECd, Dias J. Inovações Vistas pelas Patentes: Exigências Frente às Novas Funções das Universidades. Revista Brasileira de Inovação 2005; 4(2):329-62.

8. Juanola-Feliu E, Colomer-Farrarons J, Miribel-Català P, Samitier J, Valls-Pasola J. Market challenges facing academic research in commercializing nano-enabled implantable devices for in-vivo biomedical analysis. Technovation 2012; 32(3-4):193-204.

9. Lee S, Yoon B, Park Y. An approach to discovering new technology opportunities: Keyword-based patent map approach. Technovation 2009; 29(6-7):481-97.

10. Banerjee A, Hajatdoost-Sani M, Farrell S, Thompson I. A clinical evaluation and comparison of bioactive glass and sodium bicarbonate air-polishing powders. J Dent 2010; 38(6):475-9.

11. Rees JS, Addy M. A cross-sectional study of dentine hypersensitivity. J Clin Periodontol 2002; 29(11):997-1003.

12. Wichgers TG, Emert RL. Dentin hypersensitivity. Oral Health. 1997;87(3):51-3, 5-6, 9; quiz 61.

13. Yilmaz HG, Kurtulmus-Yilmaz S, Cengiz E. Long-Term Effect of Diode Laser Irradiation Compared to Sodium Fluoride Varnish in the Treatment of Dentine Hypersensitivity in Periodontal Maintenance Patients: A Randomized Controlled Clinical Study. Photomed Laser Surg 2011; 29(11):721-5.

14. Brannstrom M, Linden LA, Astrom A. The hydrodynamics of the dental tubule and of pulp fluid. A discussion of its significance in relation to dentinal sensitivity. Caries Res 1967; 1(4):310-7.

15. Castillo JL, Rivera S, Aparicio T, Lazo R, Aw TC, Mancl LL, et al. The Short-term Effects of Diammine Silver Fluoride on Tooth Sensitivity: a Randomized Controlled Trial. J Dent Res 2011; 90(2):203-8.

16. Kishore A, Mehrotra KK, Saimbi CS. Effectiveness of desensitizing agents. J Endod 2002; 28(1):34-5.

17. Yilmaz HG, Cengiz E, Kurtulmus-Yilmaz S, Leblebicioglu B. Effectiveness of Er,Cr:YSGG laser on dentine hypersensitivity: a controlled clinical trial. J Clin Periodontol 2011; 38(4):341-6.

18. da Rosa WL, Lund RG, Piva E, da Silva AF. The effectiveness of current dentin desensitizing agents used to treat dental hypersensitivity: a systematic review. Quintessence Int 2013; 44(7):535-46.

19. Brannstrom M. The hydrodynamics of the dental tubule and pulp fluid: its significance in relation to dentinal sensitivity. Annu Meet Am Inst Oral Biol 1966; 23:219.

20. Brannstrom M, Lind PO. Pulpal response to early dental caries. J Dent Res 1965; 44(5):1045-50.

21. Brahmbhatt N, Bhavsar N, Sahayata V, Acharya A, Kshatriya P. A double blind controlled trial comparing three treatment modalities for dentin hypersensitivity. Med Oral Patol Oral Cir Bucal 2012; 17(3): e483-90.

22. Dababneh RH, Khouri AT, Addy M. Dentine hypersensitivity - an enigma? A review of terminology, mechanisms, aetiology and management. Br Dent J 1999; 187(11):606-11.

23. Sethna GD, Prabhuji MLV, Karthikeyan BV. Comparison of Two Different Forms of Varnishes in the Treatment of Dentine Hypersensitivity: A Subject-Blind Randomised Clinical Study. Oral Health Prev Dent 2011; 9(2):143-50. 
24. Neuhäusler P. The use of patents and informal appropriation mechanisms-Differences between sectors and among companies. Technovation 2012; 32(12):681-93.

25. Datta A, Jessup L, Reed R. Corporate Reputation and the Commercialization of Innovation: Does Reputation Match Reality, and Does Innovation Matter? Technology and Investment. 2011; 2(4):256-72.

26. Santos MdM, Coelho GM, Santos DMd, Filho LF. Prospecção de tecnologias de futuro: métodos, técnicas e abordagens. Parcerias Estratégicas. 2004; 19:190-229.

\section{Endereço para correspondência:}

Evandro Piva

Universidade Federal de Pelotas

Rua Gonçalves Chaves, 457 - Centro

96015-560 Pelotas/RS

Fone/Fax: +55-53-3222-6690

E-mail: evpiva@gmail.com

Recebido: 06/11/2013. Aceito: 06/01/2014. 